\title{
SUBSTITUTION PROPERTY FOR THE RING OF CONTINUOUS RATIONAL FUNCTIONS
}

\author{
GOULWEN FICHOU, JEAN-PHILIPPE MONNIER, RONAN QUAREZ
}

\author{
MSC 2000: 14P99, 11E25, 26C15 \\ Keywords: continuous rational function, real algebraic variety, substitution property.
}

\begin{abstract}
We study the substitution property for the ring $\mathcal{R}_{0}(V)$ of continuous rational functions on a real algebraic affine variety $V$. We show that $\mathcal{R}_{0}(V)$ satisfies a substitution property along points; moreover, when $V$ is non-singular, it satisfies also a substitution property along Puiseux arcs, which characterizes $\mathcal{R}_{0}(V)$.
\end{abstract}

\section{INTRODUCTION}

Given a real algebraic variety $V \subset \mathbb{R}^{n}$, any morphism $\phi: \mathbb{R}[V] \rightarrow \mathbb{R}$ can be seen as an evaluation morphism at a certain point $x_{0} \in V$, where $\mathbb{R}[V]$ denotes the coordinate ring of $V$. For any extension $\operatorname{ring} B$ of $\mathbb{R}[V]$ a natural question is to ask whether the evaluation morphism does extend uniquely to $B$. This question is known as the substitution property, when one considers, more generally, an evaluation morphism $\phi: \mathbb{R}[V] \rightarrow R$ into a real closed field extension $R$ of $\mathbb{R}$. Such a property gives a lot of information on the real algebra of the ring $B$ namely, one may derive Artin-Lang property, Positivstellensatz, etc.

A natural class of rings $B$ to test are the different rings of functions considered in real algebraic geometry. The larger class consists in the ring of continuous (with respect to the Euclidean topology) semi-algebraic functions on $V$, where the continuity is intended with respect to the Euclidean topology on $V$. In that situation, the substitution property is known to be true by [12. A more rigid class is given by the ring of Nash functions on $V$, namely real analytic and semi-algebraic functions. Here also the substitution property holds true (see 2, 8.5.2]).

In the present paper, we study the case of the ring $\mathcal{R}_{0}(V)$ of continuous rational functions on $V$. Continuous rational functions on a real algebraic variety $V$ are those rational functions that admit a continuous extension along their poles. They form an intermediate ring between regular functions (rational functions without real poles) and continuous semi-algebraic functions. This ring has been intensively studied in several recent works ( [4, [5], [6], [7], [8], [9], [10, [11]).

The main results of the paper are the following. We first show that $\mathcal{R}_{0}(V)$ satisfies the substitution property (Theorem 2.13), generalizing a result in 44. A natural question is then whether this property characterizes the ring of continuous rational functions. However we prove that this is not the case (Proposition 2.14). This motivates the introduction of the concept of evaluation along Puiseux arcs in section 3. More precisely, we take evaluation morphisms of the form $\phi: \mathbb{R}[V] \rightarrow \mathbb{R}\left[\left[\mathrm{t}^{1 / \mathbb{N}}\right]\right]$, where $\mathbb{R}\left[\left[\mathrm{t}^{1 / \mathbb{N}}\right]\right]_{\text {alg }}$ is the ring of Puiseux power series over $\mathbb{R}$ that are algebraic over real polynomials.

Date: June 29, 2018.

We thank Michel Coste for fruitful discussions on the topic.

The authors benefit from the support of the Centre Henri Lebesgue ANR-11-LABX-0020-01. 
Depending upon our variety $V$ is singular or not, we obtain two opposite results. Firstly, if $V$ is non-singular, then $\mathcal{R}_{0}(V)$ satisfies the substitution property along arcs and moreover this property characterizes $\mathcal{R}^{0}(V)$ (see theorem $[3.5$ ). Secondly, it exists a singular variety $V$ such that $\mathcal{R}_{0}(V)$ does not satisfy the substitution property along arcs (see theorem 4.7).

\section{Substitution on Points}

\subsection{Definition and first examples.}

Definition 2.1. Let $A$ be an $\mathbb{R}$-algebra and $B$ be an $A$-algebra. We say that $B$ has the substitution property over $A$ if, for any real closed field $R$, any morphism $A \rightarrow R$ admits one and only one lifting to $B$, namely such that the following diagram is commutative :

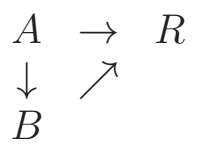

If the property if valid for the particular real closed field $R=\mathbb{R}$ we say that $B$ satisfies the weak substitution property.

Recall that an ideal $I$ of $A$ is called real if, for every sequence $a_{1}, \ldots, a_{k}$ of elements of $A$, then $a_{1}^{2}+\cdots+a_{k}^{2} \in I$ implies $a_{i} \in I$ for $i=1, \ldots, k$. A field $F$ is called real if ( 0 ) is a real ideal in $F$.

Here is the first easy fact :

Proposition 2.2. Let us assume that $A$ is a domain whose fraction field is real. If $B$ satisfy the substitution property over $A$, then the morphism $A \rightarrow B$ is injective.

Proof. Let us assume that $0 \neq a \in A$ is sent onto 0 in $B$. Since the fraction field of $A$ is real, the null ideal (0) in $A$ is real and hence it is the intersection of all the real prime ideal of $A$. Then, there is a real prime ideal $\mathfrak{p}$ in $A$ such that $a \notin \mathfrak{p}$. Take then for $R$ the real closure of the residual field at $\mathfrak{p}$. Then, $a$ is sent onto a non-zero element in $R$, which leads to a contradiction since it should be 0 after lifting the evaluation morphism to $B$.

In the following, we will mainly consider rings $B$ which are subrings of the fraction field of a domain $A$. If $f=p / q$ where $p$ and $q$ lye in $A$, starting from a morphism $\phi: A \rightarrow R$, it is easy to define $\phi(f)=\frac{\phi(p)}{\phi(q)}$ as long as $\phi(q) \neq 0$. This elementary observation gives us the substitution property if $B$ is a subring of the ring of regular functions of $A$. We recall that the ring of regular functions of $A$ is just the ring $\left(1+\sum A^{2}\right)^{-1} A$.

Hence, in all the following, we will mainly consider rings $B$ which are subrings of the field of rational functions and which contain the ring of regular functions of $A$. And the problem we will face is to define $\phi(f)$ when $\phi(q)=0$.

The first important consequence of definition 2.1, is that one gets a one-to-one correspondence between the real spectrum of $B$ and $A$. For background about the real spectrum of a ring, we refer to [2]. In few words, let us say that a point $\alpha$ of the real spectrum of the ring $A$ is an equivalence class of morphisms $\pi$ from $A$ into a real closed field for the equivalence relation generated by the following : $\pi \sim \pi^{\prime}$ where $\pi: A \rightarrow R$ and $\pi^{\prime}: A \rightarrow R^{\prime}$ with $R$ and $R^{\prime}$ are real closed field, if there exists a factorization of $\pi^{\prime}$ through a morphism $R \rightarrow R^{\prime}$.

The real spectrum is endowed with two natural topologies : the real spectrum topology and the constructible topology which are defined as follows. Any element $a \in A$ can be evaluated at any $\alpha \in \operatorname{Spec}_{r} A$ simply by evaluating it at the morphism $\pi: A \rightarrow R$ given by $\alpha$. Then, 
$\left\{\alpha \in \operatorname{Spec}_{r} A \mid a(\alpha)>0\right\}$ gives a basis of open neighborhood for the real spectrum topology. Then, the constructible open subsets are those obtained by boolean combination from the open subsets with respect to the real spectrum topology.

To any semi-algebraic subset $S$ of $\mathbb{R}^{n}$ is canonically associated a constructible subset $\widetilde{S}$ of Spec $_{r} \mathbb{R}\left[x_{1}, \ldots, x_{n}\right]$. This association is compatible with the boolean operations (intersection, union, complementary) and is such that the semi-algebraic subset $S=\left\{x \in \mathbb{R}^{n} \mid p(x)>0\right\}$ where $p \in \mathbb{R}\left[x_{1}, \ldots, x_{n}\right]$ is associated to $\widetilde{S}=\left\{\alpha \in \operatorname{Spec}_{r} \mathbb{R}\left[x_{1}, \ldots, x_{n}\right] \mid p(\alpha)>0\right\}$.

Now we state the announced correspondence.

Proposition 2.3. If $B$ satisfy the substitution property over $A$, then the induced morphism on the real spectrum $\mathrm{Spec}_{r} B \rightarrow \mathrm{Spec}_{r} A$ is bijective and continuous (with respect to both the real spectrum topology and the constructible topology).

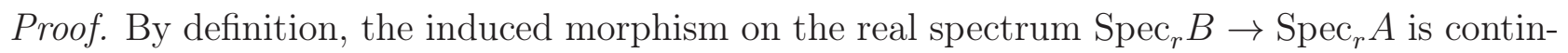
uous with respect to the real spectrum topology and also for the constructible topology (see $[2,7.17])$.

Surjectivity comes immediately from the existence of the factorization in the substitution property.

Injectivity comes immediately from the uniqueness of the factorization in the substitution property. Indeed, let us consider two points $\beta: B \rightarrow R$ and $\beta^{\prime}: B \rightarrow R^{\prime}$ where $R, R^{\prime}$ are real closed fields and assume that the compositions $\beta \circ \phi$ and $\beta^{\prime} \circ \phi$ by $\phi: A \rightarrow B$ give rise to the same point in $\operatorname{Spec}_{r} A$. Namely, there exists a real closed filed $R^{\prime \prime}$ which extends both $R$ and $R^{\prime}$, which implies that $\beta=\beta^{\prime}$.

From now on, we will takes for $A$ a ring of polynomials functions (or coordinate ring) $\mathbb{R}[V]$, or one of its localization, where $V$ is an algebraic variety in $\mathbb{R}^{n}$ in the sense of [2].

There are two well-known classes of functions whose ring satisfies the substitution property : Nash functions and continuous semi-algebraic functions.

More precisely, if $A=\mathbb{R}\left[X_{1}, \ldots, X_{n}\right]$, then one has the substitution property for the ring of Nash functions on $\mathbb{R}^{n}([2,8.5 .2])$ and one may generalize it to any smooth algebraic variety $V$ in $\mathbb{R}^{n}$.

The substitution property is also valid in the ring of semi-algebraic continuous functions on $V$. This result can be seen as a consequence of the theory of real closed rings developed by $\mathrm{N}$. Schwartz in [12].

See also the work of J. Fernando on the subject [3].

In these two cases, since a morphism $A \rightarrow R$ corresponds to the evaluation at some point $\left(x_{1}, \ldots, x_{n}\right)$ in $V \subset \mathbb{R}^{n}$, the substitution property says that any evaluation morphism can be uniquely lifted to the ring $B$.

Beware that a subring of a ring satisfying the substitution property does not necessarily satisfies the substitution property as it is shown by the following example :

Example 2.4. Let $A=\mathbb{R}[x]$ and $B=\mathbb{R}\left[x, \sqrt{1+x^{2}}\right] \simeq \mathbb{R}[x, y] /\left(y^{2}-\left(1+x^{2}\right)\right)$. Then, the morphism $A \rightarrow \mathbb{R}$ which send $x$ to 0 (the evaluation morphism at the origin) admits two liftings to $B$ : the morphism which send $y$ to 1 and the morphism which send $y$ to -1 . Moreover, $B$ is a subring of the ring of semi-algebraic continuous functions on $\mathbb{R}$.

2.2. Substitution for continuous rational functions. Let $V \subset \mathbb{R}^{n}$ be a real algebraic variety. We will mainly be interested in the ring of continuous rational functions defined on $V$. These functions are rational functions on $V$ which can be seen as functions defined on the 
complementary of the zero set of their denominators, and which admits a continuous extension to the whole $V$. In order to avoid pathological cases, we will mainly assume that the variety $V$ is central which means that the set of non-singular points of $V$ is dense in $V$ for the euclidean topology. Of course, any non-singular variety is central.

Since we require for our rings to be contained in the field of fraction $\mathbb{R}(V)$ of $V$, one should consider the natural assumption for the variety $V$ to be moreover irreducible. So let us give now the formal definition of continuous rational functions.

Definition 2.5. Let $V \subset \mathbb{R}^{n}$ be an irreducible central real algebraic variety. A continuous function $f: V \rightarrow \mathbb{R}$ is said to be continuous rational if there exists a non-empty Zariski open subset $W \subset V$ such that the restriction $f_{\mid W}$ of $f$ to $W$ is a regular function on $W$. We denote by $\mathcal{R}_{0}(V)$ the ring of continuous rational functions on $V$.

Remark 2.6. An alternative ring of functions to work with would be the ring of hereditarily continuous rational or regulous functions on a central variety $V$ defined in [5] as those continuous rational functions which remains rational in restriction to any subvariety. When the variety $V$ is smooth, we recover continuous rational functions, but in general it leads to a proper subring. We will consider this ring solely in Theorem 2.13 .

Let $A=\mathbb{R}\left[X_{1}, \ldots, X_{n}\right]$. Let us start with an elementary observation about rings of functions satisfying the substitution property. If $f$ is a real function defined on a subset of $\mathbb{R}^{n}$, we denote by $\mathcal{Z}(f)$ the zero set of $f$.

Proposition 2.7. Let $A=\mathbb{R}\left[x_{1}, \ldots, x_{n}\right]$ and $B$ be a sub A-algebra of $\mathbb{R}\left(x_{1}, \ldots, x_{n}\right)$ satisfying the substitution property over $A$.

Let $f=p / q \in B$ with $p$ and $q$ coprimes. Then,

(i) $\mathcal{Z}(q) \subset \mathcal{Z}(p)$,

(ii) $\mathcal{Z}(q)$ has codimension at least 2 in $\mathbb{R}^{n}$.

Proof. To show (i), let us just write $q=f p$ and use the substitution property for $B$ over $A$.

To show (ii), assume there exists an irreducible factor $q_{1}$ of $q$ corresponding to a codimension one variety in $\mathbb{R}^{n}$. Then, the condition $\mathcal{Z}\left(q_{1}\right) \subset \mathcal{Z}(p)$ implies that $q_{1}$ divides $p$ since $\left(q_{1}\right)$ is a real prime ideal (cf the so-called change of signs criterion [2, 4.5.1]), in contradiction with the coprimality of $p$ and $q$.

Remark 2.8. Note that in the one-dimensional case, condition (i) says already that $B$ is a subring of the ring of regular functions.

Note that the previous proposition generalizes to non-singular varieties :

Proposition 2.9. Let $A=\mathbb{R}[V]$ where $V$ is a non-singular irreducible algebraic variety in $\mathbb{R}^{n}$. Let $B$ be a sub A-algebra of $\mathbb{R}(V)$ satisfying the substitution property over $A$.

Let $f=p / q \in B$ with $p$ and $q$ coprimes i.e $(p)+(q)=1$. Then,

(i) $\mathcal{Z}(q) \subset \mathcal{Z}(p)$,

(ii) $\mathcal{Z}(q)$ has codimension at least 2 in $V$.

Proof. The coordinates ring $\mathbb{R}[V]_{m_{x}}$ of the variety $V$ localized at any point $x \in V$ is regular and hence an UFD, so that the proof can be carried out similarly.

This result can be used when $B=\mathcal{R}_{0}(V)$ where $V \subset \mathbb{R}^{n}$ is non-singular (see theorem 2.12). On the contrary, the proposition cannot be extended to the case $V$ is singular as illustrated by the example of the Cartan umbrella. 
Example 2.10. Let $V \subset \mathbb{R}^{3}$ with equation $x^{3}=z\left(x^{2}+y^{2}\right)$, and consider the rational function on $V$ given by $f=x^{3} /\left(x^{2}+y^{2}\right)$ extended by $z$ on the stick of the umbrella. Then, the zero set of its denominator $q$ is the whole stick of the umbrella, therefore of codimension one in $V$.

We come now to the central result of this section, which gives the substitution property for the ring of continuous rational functions on a non-singular real algebraic variety. We recall that the argument given in [4,5.4] relies on Eojaciewicz inequality together with the fact that a continuous rational function on $\mathbb{R}^{n}$ admits a constructible stratification such that it is regular in restriction to its strata.

This last property extend to any non-singular variety:

Theorem 2.11. Let $V \subset \mathbb{R}^{n}$ be a non-singular irreducible real algebraic variety and $f$ be a continuous rational function on $V$. Then, there exists a stratification of $V$ into Zariski locally closed subsets $S_{1}, \ldots, S_{m}$ such that the restriction $\left.f\right|_{S_{k}}$ of $f$ to $S_{k}$ is a regular function.

Proof. The proof is heavily based on the fact that, since $V$ is non-singular, $f$ is hereditarily rational (regulous) and hence one may use [10, Théorème 4.1].

Let us show now the desired substitution property for continuous rational functions:

Theorem 2.12. Let $V \subset \mathbb{R}^{n}$ be a non-singular irreducible real algebraic variety. Then, $\mathcal{R}_{0}(V)$ satisfies the substitution property over $\mathbb{R}[V]$.

Moreover, the induced morphism $\operatorname{Spec}_{r} \mathcal{R}_{0}(V) \rightarrow \operatorname{Spec}_{r} \mathbb{R}[V]$ is an homeomorphism with respect to the constructible topology.

Proof. We focus on the unicity of the factorization, since the existence comes from the usual evaluation. First, let us see what happens if $R=\mathbb{R}$. Then, one may assume, for simplicity, that the origin $o$ is in $V$ and that $\phi\left(x_{1}\right)=\ldots=\phi\left(x_{n}\right)=0$. Let $f \in \mathcal{R}_{0}(V)$. Up to considering $f-f(o)$, one may assume that $f(o)=0$.

Then, by Łojasiewicz property [2, Proposition 2.6.4] applied to $x_{1}^{2}+\ldots+x_{n}^{2}$ and $f$, there exists an integer $N$ and a continuous rational function $g$ in $\mathcal{R}_{0}(V)$ such that $f^{N}=\left(x_{1}^{2}+\ldots+x_{n}^{2}\right) g$. This algebraic identity implies that $\phi(f)=0$ which concludes the proof for the case $R=\mathbb{R}$.

Let us now consider a general real closed field $R$ and denote by $\alpha$ the point of the real spectrum of $\mathbb{R}[V]$ given by the morphism $\phi$. According to theorem 2.11 , we know that, for any $f \in \mathcal{R}_{0}(V)$, there is a Zariski locally closed stratification $V=S_{0} \cup \ldots \cup S_{m}$ such that $f$ is regular on each $S_{i}$. Let us assume that $\alpha \in \widetilde{S}_{0}$; if we set

$$
S_{0}=\{x \in V \mid r(x)=0, s(x) \neq 0\}
$$

where $r, s$ are polynomials, then $\alpha \in \widetilde{S}_{0}$ just means that $\phi(r)=0$ and $\phi(s) \neq 0$.

Then, the zero set of $r$ is contained in the zero set of $s \cdot(q f-p)$ where $p, q$ are polynomials such that $f=p / q$ is regular on $S_{0}$. Again, by Łojasiewicz property one gets the existence of an integer $N$ and a continuous rational function $g$ such that $(s(q f-p))^{N}=r g$. Applying $\phi$, and since $\phi(s) \neq 0$ and $\phi(q) \neq 0$ (since $p / q$ is regular on $S_{0}$ ), one gets $\phi(f)=\phi(p) / \phi(q)$ which concludes the first part of the proof.

By proposition $\left[2.3\right.$, the map $\varphi: \operatorname{Spec}_{r} \mathcal{R}_{0}(V) \rightarrow \operatorname{Spec}_{r} \mathbb{R}[V]$ is continuous and bijective. Let us consider a constructible set $T$ in $\operatorname{Spec}_{r} \mathcal{R}_{0}(V)$ which is a boolean combination of sets of the form $S=\{f=0, g>0\}$ where $f, g \in \mathcal{R}_{0}(V)$. Again by theorem 2.11, one knows that there is a Zariski locally closed stratification $V=S_{0} \cup \ldots \cup S_{m}$ such that $f$ and $g$ are regular on each $S_{k}$. Namely, one may write on $S_{k}, f=p_{k} / q_{k}$ and $g=r_{k} / s_{k}$ where $p_{k}, q_{k}, r_{r}, s_{k}$ are polynomials such that $q_{k}$ and $s_{k}$ do not vanish on $S_{k}$. 
Hence, the image of $S$ by $\varphi$ is then the union of all $\widetilde{S_{k}} \cap \varphi(S)$ which can be written as the subset $\widetilde{S_{k}} \cap\left\{p_{k}=0, r_{k} s_{k}>0\right\}$ in $\operatorname{Spec}_{r} \mathbb{R}[V]$. Finally, one gets that $\varphi(T)$ is a constructible subset in $\operatorname{Spec}_{r} \mathbb{R}[V]$.

The previous proposition says that if a $\mathbb{R}$-algebra homomorphism from $\mathcal{R}_{0}(V)$ is the evaluation at a given point in restriction to the polynomials, then it is still the evaluation at this point on any continuous rational functions on $V$.

So far it does not seem very clear how to get the substitution property for the ring of continuous rational functions on a singular variety since one no longer dispose of the decomposition of rational functions as regular functions on strata.

Although, one may obtain formally, by taking some quotients in a commutative diagram, that the ring of regulous functions on a singular variety $V$ satisfies the substitution property over $\mathbb{R}[V]$. By definition, the ring of regulous functions on real algebraic variety $V \subset \mathbb{R}^{n}$, given by an ideal $I \subset \mathbb{R}\left[X_{1}, \ldots, X_{n}\right]$, is just the quotient $\operatorname{ring} \mathcal{R}^{0}(V)=\mathcal{R}_{0}\left(\mathbb{R}^{n}\right) / \mathcal{I}_{\mathcal{R}_{0}\left(\mathbb{R}^{n}\right)}(V)$ where $\mathcal{I}_{\mathcal{R}_{0}\left(\mathbb{R}^{n}\right)}(V)=\left\{f \in \mathcal{R}_{0}\left(\mathbb{R}^{n}\right) \mid V \subset \mathcal{Z}(f)\right\}$. Hence, one may easily derive the substitution property for the ring of regulous functions on $V$. Namely:

Theorem 2.13. Let $V \subset \mathbb{R}^{n}$ be a central irreducible real algebraic variety whose coordinate ring is $\mathbb{R}[V]=\mathbb{R}\left[X_{1}, \ldots, X_{n}\right] / I$. Then $\mathcal{R}^{0}(V)$ satisfies the substitution property over $\mathbb{R}[V]$.

Proof. Let $A=\mathbb{R}\left[X_{1}, \ldots, X_{n}\right], B=\mathcal{R}^{0}\left(\mathbb{R}^{n}\right)$ and $\mathcal{I}=\mathcal{I}_{\mathcal{R}_{0}\left(\mathbb{R}^{n}\right)}(V)$.

The following commutative diagram is a consequence of the universal property for quotient rings :

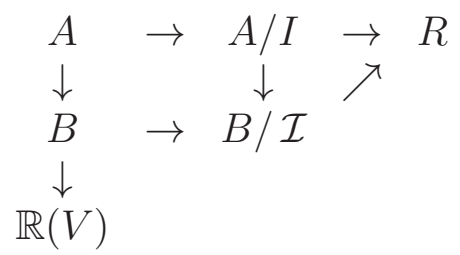

Namely, given an evaluation morphism $A / I \rightarrow R$ it gives rise to a unique morphism $A \rightarrow R$ which sends $I$ onto 0 . Hence, by the substitution property for $B$, one has an unique lifting morphism $B \rightarrow R$ which send $\mathcal{I}$ onto 0 . Hence, by the universal property for quotient rings, one has a unique factorization $B / \mathcal{I} \rightarrow R$.

\subsection{The substitution property does not characterize continuous rational functions.}

We show that the ring of continuous rational functions is not the biggest subring of rational functions that satisfies the substitution property and hence, the substitution property is not a characterization for the ring of continuous rational functions. This result will lead to the notion of substitution along arcs in the next section for which such a characterization will be available.

But for the classical substitution property, already in the case of the plane one has:

Proposition 2.14. The ring $\mathcal{R}_{0}\left(\mathbb{R}^{2}\right)$ is not maximal in $\mathbb{R}(x, y)$ to satisfy the substitution property.

Before entering into the details of the proof, we will state as an intermediary step that there exist bigger rings that satisfy only the existence condition of the lifting in the substitution property. Namely, 
Proposition 2.15. The ring $\mathcal{R}_{0}\left(\mathbb{R}^{2}\right)$ is not maximal in $\mathbb{R}(x, y)$ among rings satisfying the existence condition of the lifting in the substitution property. Moreover, there does not exist a unique maximal ring satisfying the existence condition of the lifting in the substitution property.

Proof. For the first point, let us show that the ring $\mathcal{R}_{0}\left(\mathbb{R}^{2}\right)\left[\frac{x}{x^{2}+y^{2}}\right]$ satisfies also the existence condition of the lifting in the substitution property.

It suffices to show that the evaluation morphism at the origin $\phi: \mathcal{R}_{0}\left(\mathbb{R}^{2}\right) \rightarrow \mathbb{R}$ can be lifted to $\mathcal{R}_{0}\left(\mathbb{R}^{2}\right)\left[\frac{x}{x^{2}+y^{2}}\right]$. The first step in the proof is to show that one may set $\phi\left(\frac{x}{x^{2}+y^{2}}\right)=0$.

Consider the morphism $\mathcal{R}_{0}\left(\mathbb{R}^{2}\right)[T] \stackrel{\psi}{\longrightarrow} \mathbb{R}(x, y)$ defined by $T \mapsto \frac{x}{x^{2}+y^{2}}$. One has

$$
\mathcal{R}_{0}\left(\mathbb{R}^{2}\right)\left[\frac{x}{x^{2}+y^{2}}\right] \simeq \mathcal{R}_{0}\left(\mathbb{R}^{2}\right)[T] / \operatorname{Ker} \psi
$$

Let $P \in \operatorname{Ker} \psi$, and write $P=a_{0}+\ldots+a_{n} T^{n}$ where the $a_{i}$ 's are continuous rational functions on $\mathbb{R}^{2}$. In $\mathbb{R}(x, y)[T]$, one may factorize $P$ by $T-p / q$ where $p=x, q=x^{2}+y^{2}$. Hence

$$
\frac{(q T-p)\left(b_{0}+\ldots+b_{n-1} T^{n-1}\right)}{d}=a_{0}+\ldots+a_{n} T^{n}
$$

where the $b_{i}$ 's and $d$ are polynomials in $\mathbb{R}[x, y]$.

Then,

$(*) \begin{cases}-b_{0} p & =a_{0} d \\ q b_{0}-b_{1} p & =a_{1} d \\ \cdots & \\ q b_{n-2}-b_{n-1} p & =a_{n-1} d \\ q b_{n-1} & =a_{n} d\end{cases}$

Let $r$ be the valuation of $d$ with respect to the prime $p=x$, namely $v_{p}(d)=r$. Since $p$ and $q$ are coprime, one gets from the last identity in $(*)$ that $v_{p}\left(b_{n-1}\right) \geq r$ since $(p)$ is a real prime ideal. Likewise, $v_{p}\left(b_{n-2}\right) \geq r$ and more generally $v_{p}\left(b_{i}\right) \geq r$ for $i=0, \ldots, n-1$. The first identity in $(*)$ implies that we may write $a_{0}=h / k$ with $h, k \in \mathbb{R}[x, y], v_{p}\left(a_{0}\right) \geq 1$ and $v_{p}(k)=0$. It follows that $a_{0}$ vanishes on a non-empty Zariski open subset of $\mathcal{Z}(x)$ and thus on whole $\mathcal{Z}(x)$. Hence $a_{0}(0)=0$.

Hence, the setting $\phi\left(\frac{x}{x^{2}+y^{2}}\right)=0$ is compatible with any algebraic relation verified by $f=$ $\frac{p}{q}=\frac{x}{x^{2}+y^{2}}$ over $\mathcal{R}_{0}\left(\mathbb{R}^{2}\right)$. It defines then a lifting of $\phi$ to $\mathcal{R}_{0}\left(\mathbb{R}^{2}\right)\left[\frac{x}{x^{2}+y^{2}}\right]$.

Note that, if one set $\phi_{\alpha}\left(\frac{x}{x^{2}+y^{2}}\right)=\alpha \in \mathbb{R}$, one may replace $f$ with $g=f-\alpha$, namely replace $x$ with $p=x-\alpha\left(x^{2}+y^{2}\right)$ which remains real prime. One can then repeat the previous process to get that $a_{0}(0)=0$ for any algebraic relation $a_{0}+\ldots+a_{n} g^{n}=0$ where the $a_{i}$ 's are continuous rational functions. Since $\phi_{\alpha}(g)=0$, again one has a lifting of $\phi_{\alpha}$ to $\mathcal{R}_{0}\left(\mathbb{R}^{2}\right)\left[\frac{x}{x^{2}+y^{2}}\right]$. Hence, one has infinitely many liftings of $\phi$ to $\mathcal{R}_{0}\left(\mathbb{R}^{2}\right)\left[\frac{x}{x^{2}+y^{2}}\right]$.

Let us show now the second point. Set $f=\frac{x}{x^{2}+y^{2}}$ and $g=\frac{y}{x^{2}+y^{2}}$. Let $B_{1}$ be a ring that contains $\mathcal{R}_{0}\left(\mathbb{R}^{2}\right)[f]$ and that satisfies the existence in the substitution property. Then $g$ cannot belong to $B_{1}$. Indeed, from the identity $x f+y g=1$ we get, using that $\phi$ is the evaluation at the origin, that

$$
1=\phi(x f+y g)=\phi(x) \phi(f)+\phi(y) \phi(g)=0 .
$$


Likewise, let $B_{2}$ be a ring that contains $\mathcal{R}_{0}\left(\mathbb{R}^{2}\right)[g]$ and that satisfies the existence in the substitution property. As previously, one has $f \notin B_{2}$.

Then, there does not exist a unique maximal ring satisfying the existence condition of the lifting in the substitution property.

The proof of Proposition 2.14 is obtained by slightly modifying the above proof.

Proof of Proposition 2.14. Let us consider the ring

$$
B=\mathcal{R}_{0}\left(\mathbb{R}^{2}\right)\left[\frac{x}{\left(x^{2}+y^{2}\right)^{n}}, n \in \mathbb{N}^{*}\right] .
$$

Setting $f_{n}=\frac{x}{\left(x^{2}+y^{2}\right)^{n}}$, one has $f_{n}=f_{n+1}\left(x^{2}+y^{2}\right)$. Let us note that it suffices to consider the

evaluation morphism at the origin $A=\mathbb{R}[x, y] \stackrel{\phi}{\rightarrow} \mathbb{R}$, and show that it admits one and only one lifting to $B$.

If such a lifting (again denoted by $\phi$ ) of the evaluation at the origin exists, it satisfies necessarily $\phi\left(f_{n}\right)=\phi\left(f_{n+1}\right) \phi\left(\left(x^{2}+y^{2}\right)\right)$. Since $\phi\left(x^{2}+y^{2}\right)=0$, it follows that $\phi\left(f_{n}\right)=0$ for any $n$. This shows the unicity of the lifting.

To show the existence, let us consider an algebraic relation of the form $P\left(f_{1}, \ldots, f_{n}\right)=0$ where $P=a_{0}+\sum_{\alpha>0} a_{\alpha} x^{\alpha_{1}} \ldots x^{\alpha_{n}}$ is a polynomial whose coefficients are continuous rational functions on $\mathbb{R}^{2}$. The relation $P\left(f_{1}, \ldots, f_{n}\right)=0$ can be rewritten as

$$
a_{0}+\sum_{\alpha>0} a_{\alpha} \frac{x^{\alpha_{1}+\ldots+\alpha_{n}}}{\left(x^{2}+y^{2}\right)^{\alpha_{1}+2 \alpha_{2}+\ldots+n \alpha_{n}}}=0
$$

which implies

$$
a_{0}\left(x^{2}+y^{2}\right)^{N}=-\sum_{\alpha>0} a_{\alpha}\left(x^{2}+y^{2}\right)^{N(\alpha)} x^{\alpha_{1}+\ldots+\alpha_{n}}
$$

where $N$ and $N(\alpha)$ are integers.

From the previous identity, it follows that $a_{0}$ vanishes on $\mathcal{Z}(x)$ and one gets $\phi\left(a_{0}\right)=0$. Hence $\phi\left(P\left(f_{1}, \ldots, f_{n}\right)\right)=0$ as desired.

In summary, the substitution property as defined in 2.1 is not strong enough to characterize continuous rational functions. More precisely, the lifting property is not sufficient to implies continuity, even if the evaluation morphism can be taken over a Puiseux series field. For instance, we may consider a rational function $f=p / q$ on the plane such that $p(0)=q(0)=$ $f(0)=0$, and an algebraic plane curve at the origin parametrized by two Puiseux series $\gamma=(\alpha(t), \beta(t))$ which can also be seen as an element of the real spectrum of $\mathbb{R}[x, y]$. If $\gamma$ goes to the origin by assumption, it seems not obvious to deduce that $f(\gamma)$ also goes to the origin.

These considerations lead us to consider a new substitution property along (convergent) arcs in the remaining of the paper.

\section{Substitution AlONG ARCS}

The ring of all formal power series over $\mathbb{R}$ in the indeterminate $t$ will be denoted by $\mathbb{R}[[t]]$. The ring of all formal power series over $\mathbb{R}$ in the indeterminate $t$ which are algebraic over $\mathbb{R}[t]$ will be denoted by $\mathbb{R}[[t]]_{\text {alg }}$.

We consider moreover several power series rings which are all subrings of the field of Puiseux power series. The field of all Puiseux power series over $\mathbb{R}$ in the indeterminate $t$ will be denoted by $\mathbb{R}\left(\left(\mathrm{t}^{1 / \mathbb{N}}\right)\right)$, and its valuation ring by $\mathbb{R}\left[\left[\mathrm{t}^{1 / \mathbb{N}}\right]\right]$. The subfield of Puiseux series which are algebraic over $\mathbb{R}[t]$ will de denoted by $\mathbb{R}\left(\left(t^{1 / \mathbb{N}}\right)\right)_{\text {alg }}$, and its valuation ring by $\mathbb{R}\left[\left[\mathrm{t}^{1 / \mathbb{N}}\right]\right]_{\text {alg }}$. From 
a geometric point of view, an element in the field $\mathbb{R}\left(\left(t^{1 / \mathbb{N}}\right)\right)_{\text {alg }}$ is identified with a continuous semi-algebraic functions germ $(0,+\infty) \rightarrow \mathbb{R}$ on the right at the origin, the $\operatorname{ring} \mathbb{R}\left[\left[\mathrm{t}^{1 / \mathbb{N}}\right]\right]_{\mathrm{alg}}$ consisting of those germs which can be extended continuously at the origin [2][Section 8.1].

We will use frequently in the sequel the following identification between arcs traced on a variety $V$, and $\mathbb{R}$-algebra morphisms $\mathbb{R}[V] \rightarrow \mathbb{R}[[t]]_{\mathrm{alg}}$. More precisely, if $V \subset \mathbb{R}^{n}$ is an algebraic variety defined by the polynomial equations $f_{1}=\ldots=f_{r}=0$, an algebraic formal arc $\gamma(t)=$ $\left(\gamma_{1}(t), \ldots, \gamma_{n}(t)\right) \in\left(\mathbb{R}[[t]]_{\mathrm{alg}}\right)^{n}$ traced on the variety $V$ satisfies $f_{1}(\gamma(t))=\ldots=f_{r}(\gamma(t))=0$. In other words, an algebraic formal arc on $V$ is given by an $\mathbb{R}$-algebra morphism $\mathbb{R}[V] \rightarrow \mathbb{R}[[t]]_{\text {alg }}$.

3.1. Definition and first properties. We state a substitution property along algebraic formal arcs and algebraic Puiseux arcs.

Definition 3.1. Let $A$ be an $\mathbb{R}$-algebra and $B$ be an $A$-algebra. We say that $B$ has the substitution property over $A$ along algebraic formal arcs (respectively algebraic Puiseux arcs) if any morphism $A \rightarrow \mathbb{R}[[t]]_{\text {alg }}\left(\right.$ resp. $\mathbb{R}\left[\left[\mathrm{t}^{1 / \mathbb{N}}\right]\right]_{\text {alg }}$ ) admits one and only one lifting to $B$.

Again, we mainly consider the case where $A$ is a ring of polynomials functions. One has the substitution property along algebraic Puiseux arcs for the ring of Nash functions and the ring of continuous semi-algebraic functions.

Proposition 3.2. Let $A=\mathbb{R}[V]$ be the coordinate ring of an non-singular real algebraic variety $V \subset \mathbb{R}^{n}$. Let $B$ be either the ring of semi-algebraic functions on $V$ or the ring of Nash functions on $V$. Then $B$ satisfies the substitution property along Puiseux arcs.

Proof. First of all, note that since all the possible rings $B$ being subrings of the ring of semialgebraic continuous functions on $V$, the evaluation along an arc given by $A \rightarrow \mathbb{R}\left[\left[\mathrm{t}^{1 / \mathbb{N}}\right]\right]_{\text {alg }}$ can always be lifted to $B$ by considering the usual composition of semi-algebraic arcs.

To prove the uniqueness, recall that $\mathbb{R}\left[\left[\mathrm{t}^{1 / \mathbb{N}}\right]\right]_{\text {alg }}$ is included into the field of algebraic Puiseux series $\mathbb{R}\left(\left(t^{1 / \mathbb{N}}\right)\right)$ which is a real closed field. Then, let us use the fact that the $\operatorname{ring} B$ satisfies the substitution property on points (as it has been recalled in subsection 2.1). Since the composite morphism $A \rightarrow \mathbb{R}\left[\left[\mathrm{t}^{1 / \mathbb{N}}\right]\right]_{\mathrm{alg}} \rightarrow \mathbb{R}\left(\left(\mathrm{t}^{1 / \mathbb{N}}\right)\right)_{\text {alg }}$ admits a unique lifting to $B$, so it is the case for a given morphism $A \rightarrow \mathbb{R}\left[\left[\mathrm{t}^{1 / \mathbb{N}}\right]\right]_{\text {alg }}$.

In the sequel, we will mainly consider rings $B$ which are subrings of the field of rational functions of an irreducible variety, and also rings containing the ring of regular functions (since those later obviously satisfies the substitution property over arcs).

Proposition 3.3. If $B$ satisfies the substitution property along algebraic formal or Puiseux arcs over $A$, then $B$ satisfies also the weak substitution property on points over $A$.

Proof. It suffices to compose with the evaluation morphism $\mathbb{R}[[t]]_{\text {alg }} \rightarrow \mathbb{R}$ (respectively with $\left.\mathbb{R}\left[\left[\mathrm{t}^{1 / \mathbb{N}}\right]\right]_{\text {alg }} \rightarrow \mathbb{R}\right)$ of an arc at its origin, by sending $t$ onto 0 .

Let us also mention an example where the weak substitution on points does not imply the substitution along arcs.

Example 3.4. Let $A=(\mathbb{R}[x])_{(x)}$ and $B=\left(\mathbb{R}[x, y] /\left(x^{2}-y^{2}\right)\right)_{(x, y)}$. Then, $B$ has the weak substitution property along points over $A$ but does not satisfy the substitution property over $A$ along arcs. Indeed, the $\operatorname{arc} x=t$ written on $A$ can be lifted to two different $\operatorname{arcs} \gamma_{1}=(t, t)$ and $\gamma_{2}=(t,-t)$ on $B$. Moreover, there is only one morphism $A \rightarrow \mathbb{R}:$ the one which sends $x$ to 0 . This morphism admits one and only one lifting to $B$, namely the morphism given by $x \mapsto 0$ and $y \mapsto 0$. 
3.2. Substitution along arcs for continuous rational functions. We show that the ring of continuous rational functions on a non-singular algebraic variety satisfies the substitution property along arcs. Moreover, we show that it is the biggest ring contained in the field of rational functions that one can expect to satisfy this property.

In other words, the substitution property along arcs characterizes, on non-singular varieties, continuous rational functions. It enlightens the importance of substitution along arcs for hereditarily rational functions, as it has already be pointed out in the work of [9] for instance.

Theorem 3.5. Let $A=\mathbb{R}[V]$ be the coordinate ring of a non-singular irreducible real algebraic variety $V \subset \mathbb{R}^{n}$. Then the ring $\mathcal{R}_{0}(V)$ of continuous rational functions on $V$ satisfies the substitution property along arcs. Moreover, if $B$ is a subring of the field $\mathbb{R}(V)$ of rational functions on $V$ which satisfies the substitution property along arcs, then $B \subset \mathcal{R}_{0}(V)$.

Proof. First of all, the natural lifting given by the evaluation of a continuous semi-algebraic function along a semi-algebraic arc gives the existence property. For the unicity, remark that $\mathcal{R}_{0}\left(\mathbb{R}^{n}\right)$ satisfies the substitution property on points (theorem 2.13). Then, any morphism $\gamma: A \rightarrow R$, where $R=\mathbb{R}\left[\left[\mathrm{t}^{1 / \mathbb{N}}\right]\right]_{\text {alg }}$ or $\mathbb{R}[[t]]_{\text {alg }}$, gives rise to a morphism $A \rightarrow \mathbb{R}\left(\left(\mathrm{t}^{1 / \mathbb{N}}\right)\right)_{\text {alg }}$ which can uniquely be lifted to $\mathcal{R}_{0}\left(\mathbb{R}^{n}\right)$. This shows the unicity for our desired substitution property, namely there cannot exist another lifting than the natural one.

Let us consider now a ring $B$ satisfying the substitution property along arcs and let us show the inclusion $B \subset \mathcal{R}_{0}(V)$.

Let us first consider the case $R=\mathbb{R}\left[\left[\mathrm{t}^{1 / \mathbb{N}}\right]\right]_{\mathrm{alg}}$. One may use the classical fact (see for instance [13. Chap 6, Lemma 4.2]) that a semi-algebraic function $f$ is continuous on $S$ if and only if $f \circ \gamma$ is continuous for all algebraic Puiseux arc $\gamma$ supported in $S$.

In fact, it is possible to adapt a little bit this result for our purpose :

Lemma 3.6. Let $f: S \rightarrow \mathbb{R}$ be a semi-algebraic function where $S$ is a semi-algebraic subset which has dimension $n$ at the point $x_{0} \in S$. Assume that $x_{0} \in T$ where $T$ is a semi-algebraic subset of dimension $<n$. Then, $f$ is continuous at $x_{0}$ if and only if $f \circ \gamma$ is continuous at 0 for any algebraic Puiseux arc $\gamma$ supported in $S \backslash T \cup\left\{x_{0}\right\}$ and passing through $x_{0}$.

Proof. If $f$ is not continuous at $x_{0}$, then there is $\epsilon>0$ such that the set

$$
\left\{\left\|x-x_{0}\right\||x \in S,| f(x)-f\left(x_{0}\right) \mid \geq \epsilon\right\}
$$

contains arbitrarily positive elements. Up to re-sizing this $\epsilon$, using the density of $S \backslash T$ in $T$, one gets that

$$
\left\{\left\|x-x_{0}\right\|\left|x \in S \backslash T \cup\left\{x_{0}\right\},\right| f(x)-f\left(x_{0}\right) \mid \geq \epsilon\right\}
$$

contains also arbitrarily positive elements. Since this set is semi-algebraic, it contains an interval $I$ with endpoints 0 . Now, by the curve selection lemma, there is an algebraic Puiseux arc $\gamma: I \rightarrow S \backslash T \cup\left\{x_{0}\right\}$ such that $\left\|\gamma(t)-x_{0}\right\|<\epsilon$ and $\left|f(\gamma(t))-f\left(x_{0}\right)\right| \geq \epsilon$. This concludes the proof.

Let $f=p / q$ in $B$ and let $\gamma$ be an algebraic Puiseux arc well-defined at the origin, which is not contained into the polar locus of $f$, namely such that $q \circ \gamma \neq 0$. Then, $f \circ \gamma=p \circ \gamma / q \circ \gamma$ is well defined in $\mathbb{R}\left(\left(\mathrm{t}^{1 / \mathbb{N}}\right)\right)_{\text {alg}}$, and necessarily $p \circ \gamma / q \circ \gamma$ has a non-negative valuation. Hence, $f \circ \gamma$ is continuous.

Moreover, using the substitution property at the origin of the arc, one deduces that the limit is $f(\gamma(0))$. In conclusion, $f \in \mathcal{R}_{0}(V)$ and hence $B \subset \mathcal{R}_{0}(V)$. 
Let us consider now the case $R=\mathbb{R}[[t]]_{\text {alg. }}$. Let $f \in B$ and as previously $\gamma$ be an algebraic Puiseux arc, well-defined at the origin, which is not contained in the polar locus of $f$. There exist an integer $m$ such that $\delta(t)=\gamma\left(t^{m}\right) \in\left(\mathbb{R}[[t]]_{\text {alg }}\right)^{n}$. Then, since $B$ satisfies the substitution property along arcs for $R=\mathbb{R}[[t]]_{\text {alg }}$, the arc $\delta$ uniquely lifts to $B$ and $f \circ \delta$ is continuous and admits $f(\delta(0))$ as a limit. Composing with the continuous semi-algebraic function $t \mapsto \sqrt[m]{t}$ on one gets that $f \circ \gamma$ is continuous and admits $f(\gamma(0))$ as a limit too.

One natural question is what happens if we replace algebraic Puiseux arcs with formal Puiseux series. It happens that the substitution property remains true for continuous rational functions.

Theorem 3.7. Let $A=\mathbb{R}[V]$ be the coordinate ring of a non-singular irreducible real algebraic variety $V \subset \mathbb{R}^{n}$. Then the ring $\mathcal{R}_{0}(V)$ of continuous rational functions on $V$ satisfies the substitution property along arcs in $\mathbb{R}\left[\left[\mathrm{t}^{1 / \mathbb{N}}\right]\right]$.

Moreover, if $B$ is a subring of the field $\mathbb{R}(V)$ of rational functions on $V$ which satisfies the substitution property along arcs in $\mathbb{R}\left[\left[\mathrm{t}^{1 / \mathbb{N}}\right]\right]$, then $B \subset \mathcal{R}_{0}(V)$.

Proof. Let us consider $\gamma: \mathbb{R}[V] \rightarrow \mathbb{R}\left[\left[\mathrm{t}^{1 / \mathbb{N}}\right]\right]$. One may see $\gamma$ as a morphism into $\mathbb{R}\left(\left(\mathrm{t}^{1 / \mathbb{N}}\right)\right)$ which is a real closed field. Then, since $\mathcal{R}_{0}(V)$ satisfies the substitution property over $\mathbb{R}[V]$ (theorem 2.12), one gets the unicity for a lifting of $\gamma$ to $\mathcal{R}_{0}(V)$.

In order to prove the existence of such a lifting, we show that the unique lifting of $\gamma$ with values in $\mathbb{R}\left(\left(\mathrm{t}^{1 / \mathbb{N}}\right)\right)$ has, in fact, values in $\mathbb{R}\left[\left[\mathrm{t}^{1 / \mathbb{N}}\right]\right]$. By the contrary, let us assume that there is a continuous rational function $f$ such that $\gamma(f) \notin \mathbb{R}\left[\left[\mathrm{t}^{1 / \mathbb{N}}\right]\right]$. By theorem [2.11, there is a Zariski locally closed stratification $V=S_{1} \cup \ldots \cup S_{t}$ such that $f$ is regular with restriction to each stratum. One derives a constructible stratification of the real spectrum of $\mathbb{R}[V]$, namely $\widetilde{V}=\widetilde{S}_{1} \cup \ldots \cup \widetilde{S_{t}}$. Moreover, the morphism $\gamma$ defines a point of $\widetilde{V}$ that we assume to lye in $\widetilde{S}_{1}$. Since $f$ is regular by stratum, there is two polynomials $p, q$ in $\mathbb{R}[V]$ such that $f=p / q$ is regular with restriction to $S_{1}$. In particular $\gamma(q) \neq 0$ and we get $\gamma(f)=\frac{\gamma(p)}{\gamma(q)}$.

The condition $\gamma(f) \notin \mathbb{R}\left[\left[\mathrm{t}^{1 / \mathbb{N}}\right]\right]$ says that $\operatorname{ord}_{t}(\gamma(p))<\operatorname{ord}_{t}(\gamma(q))$. By the Artin approximation theorem ([1]), there exists $\delta: \mathbb{R}[V] \rightarrow \mathbb{R}\left[\left[\mathrm{t}^{1 / \mathbb{N}}\right]\right]_{\text {alg }}$ that approximate $\gamma$ as close as desired for the (t)-adic topology. More precisely, if $S_{1}=\{r=0, s \neq 0\}$, where $r, s$ are two polynomials, one may choose $\delta$ satisfying

$$
\begin{aligned}
& \delta(r)=0, \\
& \operatorname{ord}_{t}(\gamma(p))=\operatorname{ord}_{t}(\delta(p)), \\
& \operatorname{ord}_{t}(\gamma(q))=\operatorname{ord}_{t}(\delta(q)), \\
& \operatorname{ord}_{t}(\gamma(s))=\operatorname{ord}_{t}(\delta(s))
\end{aligned}
$$

Then, one deduces that $\operatorname{ord}_{t}(\delta(p))<\operatorname{ord}_{t}(\delta(q))$ and $\delta \in \widetilde{S}_{1}$. Thus, $\delta(f)=\frac{\delta(p)}{\delta(q)}$. By theorem 3.5, one knows that $\delta(f) \in \mathbb{R}\left[\left[\mathrm{t}^{1 / \mathbb{N}}\right]\right]_{\text {alg }}$ and hence $\operatorname{ord}_{t}(\gamma(p)) \geq \operatorname{ord}_{t}(\gamma(q))$, a contradiction. This concludes the proof.

Remark 3.8. The proof of Theorem 3.7 heavily relies on the existence of a stratification, associated with a given continuous rational function, such that the function is regular on the strata. This result can also be generalized (with exactly the same proof) to a singular real algebraic variety if we replace the ring of continuous rational functions by the ring of regulous functions as considered in Theorem 2.13. Moreover, all the previous results that concern nonsingular real algebraic varieties can be extended to central real algebraic varieties with isolated singularities since in this case rational continuous functions are still regulous [11, Theorem 
2.25]. However, the question to know whether we get the same notion if we replace algebraic Puiseux arcs with formal ones in the definition of the substitution along arcs for general rings (definition 3.1) seems open.

Another natural question which arises is what can be said about substitution along arcs for continuous rational functions defined on singular varieties. Note already that this times considering Puiseux arcs or formal arcs makes a crucial difference.

Example 3.9. Consider the (singular but central) variety $V \subset \mathbb{R}^{3}$ defined by the equation $y^{3}=z^{2} x^{3}$. The arc $\gamma$ given by $x=y=0$ and $z=t$ is contained in $V$. Let us now consider the rational function $f=y / x$ on $V$. This function is continuous on $V$ because of the relation $f^{3}=z^{2}$. Note now that the power series arc $\gamma \in\left(\mathbb{R}[[t]]_{\text {alg }}\right)^{3}$ lifts to $f \circ \gamma=t^{2 / 3}$ which belongs to $\mathbb{R}\left[\left[\mathrm{t}^{1 / \mathbb{N}}\right]\right]_{\text {alg }}$ but not to $\mathbb{R}[[t]]_{\text {alg }}$.

\section{Substitution PROPERTy FOR SINGUlar VARIETIES}

We give some examples and partial results under some assumptions and perform also a counterexample showing that there is no hope to get the substitution property along arcs for continuous rational functions defined on a general singular variety.

4.1. First facts. From now on, let $V \subset \mathbb{R}^{n}$ be a singular irreducible central algebraic variety. We first state an elementary result that will imply the weak substitution property.

Proposition 4.1. Let $A=\mathbb{R}[V]$ the coordinate ring of $V \subset \mathbb{R}^{n}$ and $B$ an A-algebra which is a subring of the ring of semi-algebraic continuous functions on $V$. Assume that $B$ satisfies the Eojasiewicz property over $A$. Namely for any $f$ and $g$ in $B$ such that $\mathcal{Z}(f) \subset \mathcal{Z}(g)$, there is $h \in B$ and an integer $n$ such that $g^{n}=f h$.

Then, $B$ satisfies the weak substitution property over points.

Proof. Let $\phi: A \rightarrow \mathbb{R}$ be a morphism. Since $B$ is a subring of the ring of semi-algebraic continuous functions, $\phi$ admits a canonical lifting to $B$. Hence, one has only to show the unicity. The argument (already met in the proof of theorem 2.12) is the following.

One may assume, for simplicity, that $\phi\left(x_{1}\right)=\ldots=\phi\left(x_{n}\right)=0$. Let us assume then, for simplicity, that the origin $o$ of $\mathbb{R}^{n}$ is in $V$. Let $f \in B$. Up to considering $f-f(o)$, one may assume that $f(o)=0$.

Then, by Łojasiewicz property applied to $x_{1}^{2}+\ldots+x_{n}^{2}$ and $f$, there exists an integer $N$ and a semi-algebraic continuous function $g \in B$ such that $f^{N}=\left(x_{1}^{2}+\ldots+x_{n}^{2}\right) g$. This algebraic identity implies that $\phi(f)=0$ which concludes the proof.

As a consequence, since the ring $\mathcal{R}_{0}(V)$ has the Łojasiewicz property, one gets :

Proposition 4.2. The ring $\mathcal{R}_{0}(V)$ of continuous rational functions on $V$ satisfies the weak substitution property on points.

One can get also the substitution along arcs but under some additional hypothesis.

Proposition 4.3. Let $\pi: \widetilde{V} \rightarrow V$ be a birational regular morphism with $\widetilde{V}$ non-singular, such that $\pi$ is an homeomorphism for the Euclidean topology. Then $\mathcal{R}_{0}(V)$ is isomorphic to $\mathcal{R}_{0}(\widetilde{V})$ and satisfies the substitution property along arcs. 
Proof. The canonical morphism $\mathcal{R}_{0}(V) \rightarrow \mathcal{R}_{0}(\widetilde{V})$, given by the composition by $\pi$, is an isomorphism whose inverse isomorphism is given by the composition by the continuous rational map $\pi^{-1}$ (the composition is still rational continuous because $\pi$ is birational).

We know, by theorem 3.5 , that $\mathcal{R}_{0}(\widetilde{V})$ satisfies the substitution property along arcs, since $\widetilde{V}$ is non-singular. Hence, the substitution property along arcs holds also for $\mathcal{R}_{0}(V)$. Indeed, one has to show that any arc $\gamma: \mathbb{R}[V] \rightarrow R$, where $R=\mathbb{R}\left[\left[\mathrm{t}^{1 / \mathbb{N}}\right]\right]_{\text {alg }}$ or $\mathbb{R}[[t]]_{\text {alg }}$, admits a unique lifting to $\mathbb{R}[\widetilde{V}]$. One may compose $\gamma$ with the evaluation morphism $e_{u}: R \rightarrow \mathbb{R}$ sending $t$ onto $u \in\left[0, \epsilon\left[\right.\right.$. Since $\widetilde{V} \rightarrow V$ is a bijection, the morphism $e_{u} \circ \gamma$ admits a unique lifting to $\mathbb{R}[\widetilde{V}]$ for any $u \in[0, \epsilon[$. This shows that $\gamma$ has a unique lifting to $\mathbb{R}[\widetilde{V}]$.

One may illustrate this result with the following example.

Example 4.4. Let us consider the surface with equation $x^{3}=\left(1+z^{2}\right) y^{3}$, for which continuous rational functions and regulous functions differ [5]. The blowing-up $\widetilde{V} \rightarrow V$ along the $z$-axis is an homeomorphism and hence the ring $\mathcal{R}_{0}(V)$ does satisfy the substitution property along arcs. Note moreover that for the continuous rational function $x / y$, the denominator has a zero set of codimension only one (to be compared with the smooth case given by Proposition 2.9) ).

One may generalize a bit the previous proposition in a version which will be useful in the next subsection.

Proposition 4.5. Let $W \subset \mathbb{R}^{m}$ be an irreducible real algebraic variety, $\pi: W \rightarrow V$ a surjective regular birational morphism and assume $V$ is central. Then, $\mathcal{R}_{0}(V)$ is isomorphic to the ring $\mathcal{R}_{0}^{\pi}(W)$ of continuous rational functions on $W$ which are constant on the fibers of $\pi$.

Proof. Let us consider the morphism $\pi^{*}: \mathcal{R}_{0}(V) \rightarrow \mathcal{R}_{0}^{\pi}(W)$ defined by $f \mapsto f \circ \pi$. This morphism is well defined since, being a composition of continuous functions, the function $f \circ \pi$ is also continuous, and also constant on the fibers of $\pi$ by construction. It is moreover rational since $\pi$ is birational. And the morphism $\pi^{*}$ is injective since $\pi$ est surjective.

Finally, $\pi^{*}$ is surjective since any continuous function on $W$ constant on the fibers of $\pi$ goes down to a continuous function on $V$. By birationality of $\pi$, this new function on $V$ remains rational if the function on $W$ was so.

Remark 4.6. Note that if $V$ is a central variety and $\pi: W \rightarrow V$ is a blowing-up, then $\pi$ is surjective and one may use proposition 4.5.

4.2. A counterexample. The aim of this subsection is to show that the $\operatorname{ring} \mathcal{R}_{0}(V)$ does not necessarily satisfy the substitution property along $\operatorname{arcs}$ if $V$ is singular.

Theorem 4.7. Let us denote by $V$ the central hypersurface in $\mathbb{R}^{4}$ given by the equation $x^{8}=\left(z_{1}^{2}+z_{2}^{2}\right) y^{8}$. The ring $\mathcal{R}_{0}(V)$ of continuous rational functions on $V$ does not satisfy the substitution property along arcs.

Proof. We are going to prove that the unicity in the substitution fails. First note that the variety $V$ is central, irreducible and singular along the plane of coordinates $\left(z_{1}, z_{2}\right)$ and also along the $y$ axis.

Let us remark that the rational function $x^{2} / y^{2}$, which is well defined outside the singular plane, does extend by continuity on the whole $V$ to the function $\sqrt[4]{z_{1}^{2}+z_{2}^{2}}$, and hence $x^{2} / y^{2}$ defines a function $g$ in $\mathcal{R}_{0}(V)$. Note that it is not the case for the function $x / y$. Indeed, for 
all $(x, y, 1,0) \in V$, one has $\left(\frac{x}{y}\right)^{8}=1$ and hence $\frac{x}{y} \rightarrow \pm 1$ as $(x, y) \rightarrow(0,0)$. Since $x$ and $y$ can take positive and negative values on $X, \frac{x}{y}$ does not admit a limit as $(x, y)$ goes to $(0,0)$.

The blowing-up $\pi: W \rightarrow V$ of $V$ along the plane of coordinates $\left(z_{1}, z_{2}\right)$ can be seen in the chart given by the coordinates

$$
x=u v, y=v, z_{1}=w_{1}, z_{2}=w_{2}
$$

as the variety with equation $u^{8}=w_{1}^{2}+w_{2}^{2}$. Hence $W$ is the product of a surface $S$ in $\mathbb{R}^{3}$, defined by the same equation as the one of $W$, with a line (the $v$-axis). The surface $S$ has an isolated singularity at the origin, hence the singular locus of $W$ consists of the $v$-axis.

Since $V$ is a central variety, the blowing-up $\pi$ is surjective (see remark 4.6) and, using 4.5, one may identify $\mathcal{R}_{0}(V)$ with the ring $\mathcal{R}_{0}^{\pi}(W)$ of continuous rational functions on $W$ which are constant on the fibers of $\pi$.

We are going to exhibit an algebraic Puiseux arc contained in the variety $V$ which can be lifted to the ring $\mathcal{R}_{0}(V)$ in two different ways. Let us denote by $\gamma:[0, \epsilon[\rightarrow V$ the polynomial arc defined by $\gamma(t)=(0,0,0, t)$. The arc $\gamma$ is contained in the singular plane of $V$. Moreover, it can be lifted to $W$ into exactly two different algebraic Puiseux arcs given by

$$
\tilde{\gamma}_{ \pm}(t)=\left( \pm^{4} \sqrt{t}, 0,0, t\right)
$$

Lemma 4.8. Let $f \in \mathcal{R}_{0}(V)$. The semi-algebraic function $t \mapsto f \circ \gamma(t)$ is the composition of a one variable rational function $F$, regular at any point in $[0,+\infty)$, with the square root function, i.e. $(f \circ \gamma)(t)=\frac{U(\sqrt{t})}{V(\sqrt{t})}$ where $U$ and $V$ are one-variable polynomials such that $V(0) \neq 0$.

Proof. It suffices to show the result for the function $f \circ \pi$ composed with a lifting of the arc $\gamma$ on $Y$ (for instance $\tilde{\gamma}_{+}$) since $f \circ \gamma=f \circ \pi \circ \tilde{\gamma}_{+}$.

We want to restrict the function $f \circ \pi$, which is continuous rational on $W$, to the image of $\tilde{\gamma}_{+}$. The Zariski closure of the image of $\tilde{\gamma}_{+}$is the nonsingular irreducible curve $C$ defined by $u^{4}=w_{2}$ and $v=w_{1}=0$ in $S \times\{0\} \subset W$. Note that $C$ is not contained in the singular locus of $W$. Therefore the restriction of $f \circ \pi$ to $C$ is still rational by [5, Proposition 8], and of course continuous. In particular, it is a regular function on $C$ because $C$ is a nonsingular curve.

Parametrizing $C$ with $\theta \mapsto\left(\theta, \theta^{4}\right)$, one gets the existence of a regular function $G$ on $\mathbb{R}$ such that $f \circ \pi\left(\theta, 0,0, \theta^{4}\right)=G(\theta)$. Moreover, this regular function is even since $\left(\theta, 0,0, \theta^{4}\right) \in W$ and $\left(-\theta, 0,0, \theta^{4}\right) \in W$ lies over the same point $\left(0,0,0, \theta^{4}\right) \in V$ and $f \in \mathcal{R}_{\pi}^{0}(W)$. Hence, we claim there is a one-variable rational function $F$, regular at any point in $[0,+\infty)$, such that $G(\theta)=F\left(\theta^{2}\right)$. Indeed, we may write $G(\theta)=R(\theta) /(1+Q(\theta))$ with $R, Q$ some real onevariable polynomials and such that $Q$ is a sum of squares of one-variable polynomials. Since $G(\theta)=(R(\theta)(1+Q(-\theta))) /((1+Q(\theta))(1+Q(-\theta)))$ and $((1+Q(\theta))(1+Q(-\theta)))$ have even parity then it follows that $(R(\theta)(1+Q(-\theta)))$ has also even parity and we get the claim. To end, one sets $\theta^{4}=t$ and gets

$$
(f \circ \gamma)(t)=G(\sqrt[4]{t})=F(\sqrt{t})
$$

as desired.

Lemma 4.9. Any morphism $\phi: \mathcal{R}_{0}(V) \rightarrow \mathbb{R}\left[\left[\mathrm{t}^{1 / \mathbb{N}}\right]\right]_{\text {alg }}$ which extends the morphism given by the evaluation along $\gamma$ on polynomial functions, is entirely determined by the image of the continuous rational function $g$ (which extends $x^{2} / y^{2}$ by continuity on all $V$ ).

Proof. According to lemma 4.8 and since $(g \circ \gamma)(t)=\sqrt{t}$, for any $f \in \mathcal{R}_{0}(V)$, there is a onevariable rational function $F$ regular at any point in $[0,+\infty)$ such that $f \circ \gamma=F(g \circ \gamma)$. Hence, 
for any $t \in\left[0, \epsilon\left[\right.\right.$, one has $(f-F \circ g)(\gamma(t))=0$. Note also that $F \circ g \in \mathcal{R}_{0}(V)$. Since the image of $\gamma$ is locally contained in the zero set of $x^{2}+y^{2}+z_{1}^{2}$, one has, by Eojaciewicz equality ([2, théorème 2.6.6]), the existence of an integer $N$ and a semi-algebraic continuous function $h$ such that

$$
(f-F \circ g)^{N}=\left(x^{2}+y^{2}+z_{1}^{2}\right) h .
$$

Note that this equality shows also that $h \in \mathcal{R}_{0}(V)$. Hence, for any given morphism $\phi$, one has $\phi\left((f-F \circ g)^{N}\right)=0$ which gives $\phi(f)=\phi(F \circ g)$. Since $F$ is regular at 0 , one may evaluate its denominator at any arc going through the origin, and hence one gets also $\phi(f)=F(\phi(g))$.

In particular, one deduces that there exist at most two candidates for such morphisms : either $\phi_{+}$which sends $g$ onto $\sqrt{t}$ or $\phi_{-}$which sends $g$ onto $-\sqrt{t}$. The morphism $\phi_{+}$is the natural morphism coming from the evaluation of continuous semi-algebraic functions along $\gamma$. The next lemma states that the choice $\phi_{-}$is also possible.

Lemma 4.10. The setting $\phi_{-}(g)=-\sqrt{t}$ defines a morphism $\phi_{-}: \mathcal{R}_{0}(V) \rightarrow \mathbb{R}\left[\left[\mathrm{t}^{1 / \mathbb{N}}\right]\right]_{\text {alg }}$ which extends the morphism given by $\gamma$ on $\mathbb{R}[V]$ the ring of polynomial functions over $V$.

Proof. One has to check that $\phi_{-}$does respect algebraic relations existing for elements of $\mathcal{R}_{0}(V)$ over $\mathbb{R}[V]$, the ring of polynomial functions over $V$. Let $f_{1}, \ldots, f_{n}$ in $\mathcal{R}_{0}(V)$ such that $P\left(f_{1}, \ldots, f_{n}\right)=0$ where $P \in \mathbb{R}[V]$. One has to show that $\phi_{-}\left(P\left(f_{1}, \ldots, f_{n}\right)\right)=0$.

According to the end of the proof of the previous lemma, there exists a one-variable rational function $F$, regular at 0 , such that $\phi_{+}\left(P\left(f_{1}, \ldots, f_{n}\right)\right)=F\left(\phi_{+}(g)\right)$ and $\phi_{-}\left(P\left(f_{1}, \ldots, f_{n}\right)\right)=$ $F\left(\phi_{-}(g)\right)$.

Let us write $F=U / V$ where $U$ and $V$ are one-variable polynomials.

Since the natural morphism $\phi_{+}$is well defined, one gets from $P\left(f_{1}, \ldots, f_{n}\right)=0$ that $\phi_{+}\left(P\left(f_{1}, \ldots, f_{n}\right)\right)=0$. Namely, one has $F\left(\phi_{+}(g)\right)=0$, which means that $U\left(\phi_{+}(g)\right)=0$.

One may write $U(x)=S\left(x^{2}\right)+x T\left(x^{2}\right)$ where $S$ and $T$ are one-variable polynomials. Then, $U(t)=0$ for any $t \in[0, \sqrt{\epsilon}[$, and hence $S=T=0$, i.e. $U=0$.

Consequently, $F\left(\phi_{-}(g)\right)=0$ and hence $\phi_{-}\left(P\left(f_{1}, \ldots, f_{n}\right)\right)=0$, the desired equality.

As a consequence the unicity in the substitution property fails.

\section{REFERENCES}

[1] M. Artin, On the solutions of analytic equations, Invent. Math., 5 (1968) 277-291

[2] J. Bochnak, M. Coste, M.-F. Roy, Real algebraic geometry, Springer, (1998)

[3] J.F. Fernando, On the sustitution theorem for rings of semi-algebraic functions, J. Inst. Math. Jussieu $14,857-894(2015)$

[4] G. Fichou, J-P. Monnier, R. Quarez, Continuous functions on the plane regular after one blowing-up, Math. Z. 285, no. 1-2, 287-323. (2017)

[5] J. Kollár, K. Nowak, Continuous rational functions on real and p-adic varieties, Math. Z. 279, 1-2, 85-97 (2015).

[6] G. Kreisel, Review of Ershov, Zbl. 374, 02027 (1978).

[7] W. Kucharz, Rational maps in real algebraic geometry, Adv. Geom. 9 (4), 517-539, (2009)

[8] W. Kucharz, K. Kurdyka, Stratified-algebraic vector bundles, J. Reine angew. Math., to appear

[9] J. Kollar, W. Kucharz, K. Kurdyka, Curve-rational functions, Math. Ann. 370 (2018), no. 1-2, 39-69

[10] G. Fichou, J. Huisman, F. Mangolte, J.-P. Monnier, Fonctions régulues, J. Reine Angew. Math. 718 (2016), 103-151.

[11] J.-P. Monnier, Semi-algebraic geometry with rational continuous functions, Math. Ann. to appear.

[12] N. Schwartz, Real closed rings, Habilitationsschrift, München (1984).

[13] L. Van Den Dries, Tame Topology and O-minimal Structures, London Math. Soc. Lecture Note 248. Cambridge Univ. Press (1998) 
Goulwen Fichou, Univ Rennes, CNRS, IRMAR - UMR 6625, F-35000 Rennes, France

E-mail address: goulwen.fichou@univ-rennes1.fr

JeAn-Philippe Monnier, LUnAM Université, LAREMA, Université D'Angers

E-mail address: jean-philippe.monnier@univ-angers.fr

Ronan Quarez, Université de Rennes, Campus de Beaulieu, 35042 Rennes Cedex, France

E-mail address: ronan.quarez@univ-rennes1.fr 